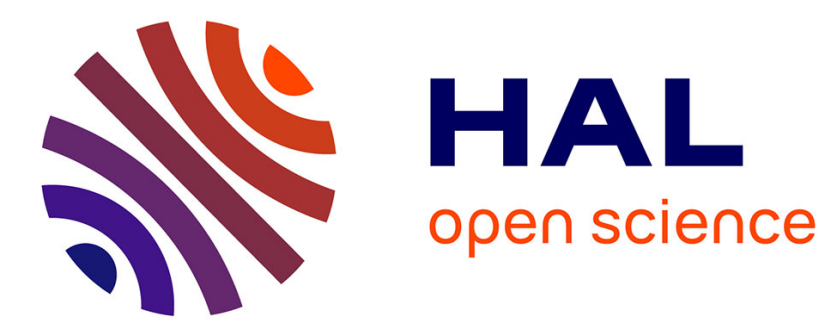

\title{
Effets paramétriques entre ondes acoustiques
}

\author{
G. Sagnes, G. Bastide
}

\section{To cite this version:}

G. Sagnes, G. Bastide. Effets paramétriques entre ondes acoustiques. Revue de Physique Appliquée, 1977, 12 (4), pp.533-541. 10.1051/rphysap:01977001204053300 . jpa-00244206

\section{HAL Id: jpa-00244206 https://hal.science/jpa-00244206}

Submitted on 1 Jan 1977

HAL is a multi-disciplinary open access archive for the deposit and dissemination of scientific research documents, whether they are published or not. The documents may come from teaching and research institutions in France or abroad, or from public or private research centers.
L'archive ouverte pluridisciplinaire HAL, est destinée au dépôt et à la diffusion de documents scientifiques de niveau recherche, publiés ou non, émanant des établissements d'enseignement et de recherche français ou étrangers, des laboratoires publics ou privés. 


\title{
EFFETS PARAMÉTRIQUES ENTRE ONDES ACOUSTIQUES
}

\author{
G. SAGNES et G. BASTIDE
}

Centre d'Etudes d'Electronique des Solides (*) Université des Sciences

et Techniques du Languedoc, place E.-Bataillon, 34060 Montpellier Cedex, France

(Reçu le 9 juin 1976, révisé le 20 décembre 1976, accepté le 22 décembre 1976)

\begin{abstract}
Résumé. - Nous rapportons les résultats d'une étude expérimentale sur CdS, de l'interaction paramétrique d'origine acoustoélectrique entre ondes de surface.

Les ondes sont du type Bleustein-Gulyaev, donc de grande profondeur de pénétration, ce qui permet de les assimiler à des ondes planes. Ces résultats sont confrontés avec ceux d'une analyse mathématique bâtie à partir de la théorie unidimensionnelle de Hutson et White sur les effets linéaires.

Nous montrons que cette analyse rend compte de façon très satisfaisante des résultats expérimentaux si on excepte les amplifications linéaires trop importantes où ni les effets de piègeage des porteurs, ni la génération d'harmoniques ne peuvent expliquer les divergences observées.
\end{abstract}

Abstract. - We report our experimental results on the parametric interaction of BleusteinGulyaev acoustic surface waves on CdS.

We compare these results with a theoretical analysis developed from the one-dimensional Hutson and White theory of linear effects.

We show that this analysis is in good agreement with the experiments except near one maximum of the linear amplification where neither trapping effects nor harmonic generation can explain the discrepancy observed.

1. Introduction. - Nous avons publié dans un précédent article [1], la mise en évidence des effets paramétriques d'origine acoustoélectrique qui se produisent lorsque 2 ondes codirectives de type Bleustein [2]-Gulyaev [3] se propagent dans le même sens dans un cristal de $\mathrm{CdS}$ photoconducteur.

Nous rapportons ici, une étude comparative entre nos résultats expérimentaux et les prévisions d'une analyse mathématique élaborée à partir de la théorie unidimensionnelle de Hutson et White [4].

La figure 1 schématise le dispositif expérimental.

La surface active des échantillons est parallèle à l'axe C. Trois paires de transducteurs interdigités accordés sur $30 \mathrm{MHz}, 40 \mathrm{MHz}$ et $70 \mathrm{MHz}$ y sont photogravés aux extrémités et permettent l'émission et la réception des ondes acoustiques.

Le champ électrique d'accélération des porteurs est appliqué soit entre deux électrodes, figure la, soit par l'intermédiaire d'une structure en segments, figure $1 \mathrm{~b}$, devant laquelle un masque mobile permet de faire varier le nombre de cellules éclairées, c'est-àdire la longueur d'interaction.

Deux trains d'ondes sont injectés à l'extrémité gauche de l'échantillon, l'un, appelé pompe avec un

(*) Laboratoire associé au C. N. R. S. niveau grand et noté $\mathrm{U}_{3}$, de $40 \mathrm{MHz}$ de fréquence et $1 \mu \mathrm{s}$ de durée, l'autre $\mathrm{U}_{2}(70 \mathrm{MHz})$, avec un niveau plus faible et $4 \mu \mathrm{s}$ de durée.

Les transducteurs de droite permettent de recevoir les trois trains d'ondes $U_{3}, U_{2}$ et $U_{1}$

$$
(30(\mathrm{MHz})=70-40) \text {. }
$$

Nous en montrons, un oscillogramme typique où $\mathrm{U}_{2}$ est affaibli dans sa partie centrale, c'est-à-dire pendant la durée de son interaction avec $\mathrm{U}_{3}$.

2. Mise en équation du problème. - Les symboles et les notations utilisés sont classiques en acoustoélectricité. Notons que les quantités caractérisant la propagation des ondes $(E, S, \rho, J, u)$ sont des quantités complexes et les figures représentent les variations du module $u$ de l'élongation $u$.

Hutson et White [4] ont montré qu'une onde acoustique plane de déformation $S$ qui se propage dans un milieu conducteur et piezoélectrique, induit un champ électrique $E$ lié à $S$ par la relation :

$$
E=-\frac{e}{\varepsilon} \frac{\gamma}{\Gamma} S
$$

où

$$
\gamma=1-\mu E_{0} / V_{\mathrm{s}} \text { et } \Gamma=\gamma+j \frac{\omega_{\mathrm{c}}}{\omega}
$$



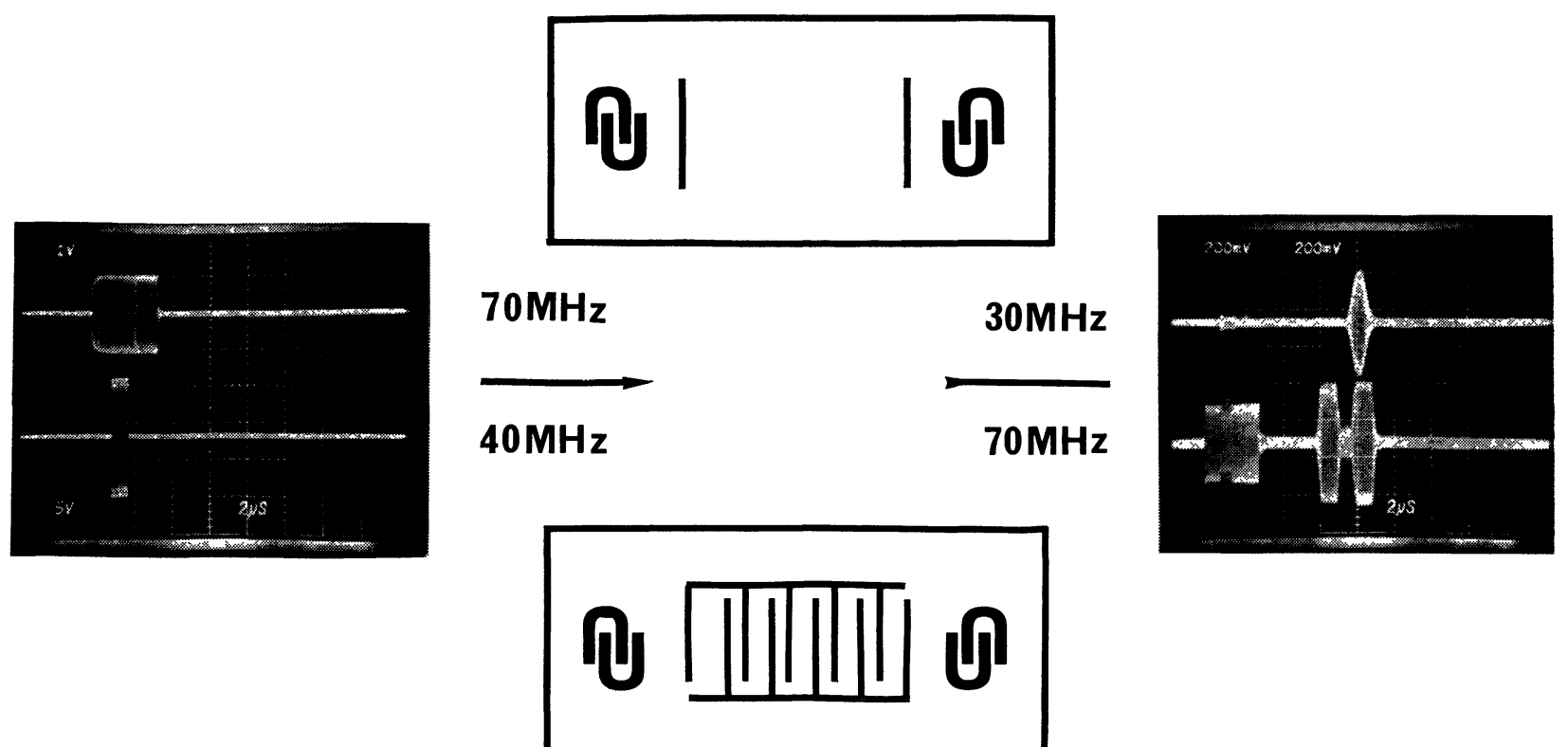

FIG. 1. - Dispositif expérimental : $a$ ) échantillon à 2 électrodes accélératrices ; $b$ ) échantillon à structure en segments : oscillogramme de gauche : signaux d'entrée $U_{30}(t)$ et $U_{20}(t)$; oscillogramme de droite : signaux de sortie $U_{1}(t)$ et $U_{2}(t)$.

Ce champ électrique module la distribution spatiale des porteurs libres en une densité :

$$
\rho=\varepsilon k \frac{\omega_{\mathrm{c}}}{\omega \gamma} E .
$$

Ces 2 grandeurs, $E$ et $\rho$, créent un courant de densité :

$$
J_{\mathrm{n} 1}=\mu \rho E
$$

qui est un terme non linéaire puisque proportionnel au carré de la déformation.

En présence de 2 ondes acoustiques de pulsations $\omega$ et $\omega^{\prime}$, il apparaît deux couples $(E, \rho)$ et $\left(E^{\prime}, \rho^{\prime}\right)$ ayant les caractéristiques de propagation de chacune des ondes.

Le courant $J_{\mathrm{n} 1}$ possède alors une composante d'expression :

$$
J=\mu\left(\rho E^{\prime}+\rho^{\prime} E\right)
$$

qui contient deux termes :

- $J_{+}$de pulsation $\omega+\omega^{\prime}$ (reconversion vers les fréquences plus élevées) et

- $J_{-}$de pulsation $\omega-\omega^{\prime}$ (reconversion vers les fréquences plus basses).

A partir de (1) et (2), on obtient [6] :

$$
\begin{aligned}
& J_{+}=\mu \omega_{\mathrm{c}} \frac{e^{2}}{\varepsilon V_{\mathrm{s}}} \frac{2 \gamma}{\Gamma \Gamma^{\prime}} S S^{\prime} \\
& J_{-}=\mu \omega_{\mathrm{c}} \frac{e^{2}}{\varepsilon V_{\mathrm{s}}} \frac{2 \gamma}{\Gamma \Gamma^{\prime *}} S S^{\prime *}
\end{aligned}
$$

où les quantités $A^{*}$ représentent les complexes conjugués des quantités $A$.

A ces 2 densités de courant correspondent deux champs électriques $E_{+}$et $E_{-}$dont les expressions s'obtiennent en développant jusqu'à l'ordre 2 l'équation de conservation du courant $\left(\operatorname{div} J+\frac{\partial \rho}{\partial t}=0\right)$ :

$$
\begin{aligned}
& E_{+}=\frac{J_{+}}{\sigma-j\left(\omega+\omega^{\prime}\right) \varepsilon} \\
& E_{-}=\frac{J_{-}}{\sigma-j\left(\omega-\omega^{\prime}\right) \varepsilon} .
\end{aligned}
$$

En combinant les expressions (5) et (6) on obtient :

$$
\begin{aligned}
& E_{+}=-j \frac{\omega_{\mathrm{c}}}{\omega+\omega^{\prime}} \frac{\mu}{V_{\mathrm{s}}} \frac{e^{2}}{\varepsilon^{2}} \frac{2 \gamma}{\Gamma \Gamma^{\prime} \Gamma_{+}} S S^{\prime} \\
& E_{-}=-j \frac{\omega_{\mathrm{c}}}{\omega-\omega^{\prime}} \frac{\mu}{V_{\mathrm{s}}} \frac{e^{2}}{\varepsilon^{2}} \frac{2 \gamma}{\Gamma \Gamma^{\prime *} \Gamma_{-}} S S^{\prime *}
\end{aligned}
$$

avec :

et

$$
\begin{aligned}
& \Gamma_{+}=\gamma+j \omega_{\mathrm{c}} /\left(\omega+\omega^{\prime}\right) \\
& \Gamma_{-}=\gamma+j \omega_{\mathrm{c}} /\left(\omega-\omega^{\prime}\right) .
\end{aligned}
$$

Le champ électrique associé à une onde acoustique de pulsation $\omega_{0}$ est donc la somme de son propre champ piezoélectrique et de tous les champs $E_{+}$ou $E_{-}$de même pulsation $\omega_{0}$ qui sont créés par l'interaction de couples d'ondes $\omega$ et $\omega^{\prime}$ tels que :

ou

$$
\begin{aligned}
& \omega+\omega^{\prime}=\omega_{0} \\
& \omega-\omega^{\prime}=\omega_{0} .
\end{aligned}
$$

L'équation de propagation [4] d'ordre 1 :

$$
\rho \frac{\partial^{2} u}{\partial t^{2}}-c \frac{\partial^{2} u}{\partial x^{2}}=e \frac{\partial E}{\partial x}
$$


devient :

$$
\rho \frac{\partial^{2} u}{\partial t^{2}}-c \frac{\partial^{2} u}{\partial x^{2}}=e \frac{\partial}{\partial x}\left[E+\sum E_{+}+\sum E_{-}\right]
$$

La résolution de cette équation ne peut s'effectuer simplement que dans l'hypothèse où la vitesse de propagation n'est pas modifiée par la présence des champs électriques $\sum E_{+}$et $\sum E_{-}$. Cette hypothèse est d'ailleurs parfaitement justifiée puisque la variation relative de vitesse $\Delta V_{\mathrm{s}} / V_{\mathrm{s}}$ introduite par le champ du $1^{\circ}$ ordre $E$, n'est que de l'ordre de $K^{2} / 2=10^{-2}$, donc cette vitesse ne sera qu'infiniment peu perturbée par la présence des autres champs qui sont des termes d'ordre supérieur.

Alors le vecteur d'onde $k$ a toujours pour expression :

$$
k=\frac{\omega}{V_{\mathrm{s}}}\left(1-\frac{K^{2}}{2} \operatorname{Réel} \frac{\gamma}{\Gamma}\right) .
$$

On cherche des solutions de la forme :

$$
X=\widehat{X} \mathrm{e}^{j(k x-\omega t)}
$$

avec $\hat{X}$ variant peu sur une longueur d'onde c'est-àdire $\partial \hat{X} / \partial x \ll j k \hat{X}$.

En introduisant l'expression de $k$ dans l'équation (8) et en posant :

$$
\alpha=\frac{K^{2}}{2} \frac{\omega}{V_{s}} \operatorname{Im} \frac{\gamma}{\Gamma}
$$

$\alpha$ gain électronique [4], on obtient :

$$
\begin{aligned}
\frac{\mathrm{d} U}{\mathrm{~d} x}=-\alpha U+\frac{e}{2 c}\left(\sum E_{+}\right. & \exp \left(j \Delta k_{+} x\right)+ \\
& \left.+\sum E_{-} \exp \left(j \Delta k_{-} x\right)\right)
\end{aligned}
$$

où les

$$
\Delta k_{+}=k+k^{\prime}-k_{0} \text { et } \Delta k=k-k^{\prime}-k_{0}
$$

sont le résultat de la propagation dans le milieu dispersif.

3. Résultats. - Nous nous intéressons au cas où 2 ondes planes de pulsations $\omega_{2}$ et $\omega_{3}$ sont en interaction dans le milieu. Elles créent par effet non linéaire 2 ondes de fréquences :

$$
f_{1}=\omega_{1} / 2 \pi=\left(\omega_{2}-\omega_{3}\right) / 2 \pi
$$

et

$$
f_{4}=\omega_{4} / 2 \pi=\left(\omega_{2}+\omega_{3}\right) / 2 \pi
$$

qu'il est commode de qualifier d'ondes du deuxième ordre et qui viennent perturber les ondes initiales en créant avec l'une d'elles, un champ électrique d'ordre 2 synchrone de l'autre puisque :

$$
\omega_{4}-\omega_{3}=\omega_{2}
$$

et

$$
\omega_{2}-\omega_{1}=\omega_{3} \text {. }
$$

Le problème consiste à résoudre le système suivant :

$$
\begin{aligned}
& \frac{\mathrm{d} U_{1}}{\mathrm{~d} x}=-\alpha_{1} U_{1}+\frac{e}{2 c}\left[E_{-}\left(\omega_{2}-\omega_{3}\right) \exp \left(j \Delta k_{231} x\right)\right] \\
& \frac{\mathrm{d} U_{2}}{\mathrm{~d} x}=-\alpha_{2} U_{2}+\frac{e}{2 c}\left[E_{-}\left(\omega_{4}-\omega_{3}\right) \exp \left(j \Delta k_{432} x\right)+E_{+}\left(\omega_{1}+\omega_{3}\right) \exp \left(j \Delta k_{132} x\right)\right] \\
& \frac{\mathrm{d} U_{3}}{\mathrm{~d} x}=-\alpha_{3} U_{3}+\frac{e}{2 c}\left[E_{-}\left(\omega_{2}-\omega_{1}\right) \exp \left(j \Delta k_{213} x\right)+E_{-}\left(\omega_{4}-\omega_{2}\right) \exp \left(j \Delta k_{423} x\right)\right] \\
& \frac{\mathrm{d} U_{4}}{\mathrm{~d} x}=-\alpha_{4} U_{4}+\frac{e}{2 c}\left[E_{+}\left(\omega_{2}+\omega_{3}\right) \exp \left(j \Delta k_{234} x\right)\right] .
\end{aligned}
$$

Dans ce système d'équations les champs électriques $E_{+}$et $E_{-}$ont des expressions analogues aux expressions (7) où $S=j k U$ et $S^{*}=-j k U^{\prime *}$.

Les conditions aux limites sont:

$$
\begin{aligned}
& U_{1}(\mathrm{O})=U_{4}(\mathrm{O})=\mathrm{O}, \quad U_{2}(\mathrm{O})=U_{20} \\
& \text { et } U_{3}(\mathrm{O})=U_{30} \text {. }
\end{aligned}
$$

3.1 Solution ANALYTiQue. - Une résolution ana1ytique approchée d'un tel système d'équations a été proposée par plusieurs auteurs [6,7]. Ils se sont limités au cas où il n'y a que 3 ondes, dont une d'un niveau suffisamment grand pour ne pas être perturbée par la présence des deux autres.
Nous proposons dans un premier temps une solution analytique analogue. Elle pêche par des hypothèses restrictives mais elle a le mérite de rendre compte de façon assez satisfaisante d'un certain nombre de phénomènes observés expérimentalement.

$1^{\text {re }}$ Hypothèse. - Tout l'effet paramétrique est produit par la seule onde d'ordre 2 de fréquence $\left(\omega_{2}-\omega_{3}\right) / 2 \pi$. L'autre de fréquence $\left(\omega_{2}+\omega_{3}\right) / 2 \pi$ intervient peu parce que, par exemple, la reconversion vers les fréquences hautes est moins efficace que vers les fréquences basses.

$2^{\mathrm{e}}$ Hypothèse. - Une onde initiale se comporte 
comme une pompe, c'est-à-dire qu'elle n'est pas influencée par l'effet paramétrique. Supposons donc:

$$
U_{3}=U_{30} \mathrm{e}^{-\alpha_{3} x} \text {. }
$$

Le système (14) se réduit alors à :

$\frac{\mathrm{d} U_{1}}{\mathrm{~d} x}=-\alpha_{1} U_{1}+\eta_{1} U_{2} U_{30} \exp \left(-\alpha_{3} x+j \Delta k x\right)$
$\frac{\mathrm{d} U_{2}}{\mathrm{~d} x}=-\alpha_{2} U_{2}+\eta_{2} U_{1} U_{30} \exp \left(-\alpha_{3} x-j \Delta k x\right)$.

Dans ce système :

$$
\begin{gathered}
\Delta k=k_{2}-k_{3}-k_{1} \\
\eta_{1}=-j \mu \frac{\omega_{c}}{V_{\mathrm{s}} \omega_{1}} \frac{e^{3}}{\varepsilon^{2} c} \frac{\gamma}{\Gamma_{1} \Gamma_{2} \Gamma_{3}^{*}} k_{2} k_{3}
\end{gathered}
$$

et

$$
\eta_{2}=j \mu \frac{\omega_{\mathrm{c}}}{V_{\mathrm{s}} \omega_{2}} \frac{e^{3}}{\varepsilon^{2} c} \frac{\gamma}{\Gamma_{1} \Gamma_{2} \Gamma_{3}} k_{1} k_{3} .
$$

En éliminant une variable entre les 2 équations, on obtient l'équation différentielle en $U_{1}$ :

$$
\begin{gathered}
\frac{\mathrm{d}^{2} U_{1}}{\mathrm{~d} x^{2}}+\left(\sum_{1}^{3} \alpha_{i}-j \Delta k\right) \frac{\mathrm{d} U_{1}}{\mathrm{~d} x}+\alpha_{1} \alpha_{2}+\alpha_{1}\left(\alpha_{3}-j \Delta k\right)= \\
-\eta_{1} \eta_{2} U_{30}^{2} \exp \left(-2 \alpha_{3} x\right) U_{1}=0
\end{gathered}
$$

et une équation similaire pour $U_{2}$. Ces 2 équations s'intégrent moyennant une troisième hypothèse.

$3^{\mathrm{e}}$ Hypothèse. $-\alpha_{3} x$ et $\Delta k x$ sont très inférieurs à 1 . Ce qui signifie que le domaine de validité du calcul est limité à des longueurs d'interaction faibles ou au voisinage du seuil d'amplification $(\gamma \simeq 0)$.

Compte tenu des conditions aux limites, on obtient alors :

$$
U_{1}=\eta_{1} \frac{U_{20} U_{30}}{\Delta_{1}^{1 / 2}}\left(\exp \left(p_{1} x\right)-\exp \left(p_{1}^{\prime} x\right)\right)
$$

avec

$$
\left.\begin{array}{ll}
2 & p_{1} \\
2 & p_{1}^{\prime}
\end{array}\right\}=-\sum_{1}^{3} \alpha_{i} \pm \Delta_{1}^{1 / 2}
$$

et

$$
\Delta_{1}=\left(\sum_{1}^{3} \alpha_{i}\right)^{2}-4\left(\alpha_{1}\left(\alpha_{2}+\alpha_{3}\right)-\eta_{1} \eta_{2} U_{30}^{2}\right) .
$$

La solution de l'équation en $U_{2}$ est :

$U_{2}=\frac{U_{20}}{\Delta_{2}^{1 / 2}}\left[\left(p_{2}+\alpha_{2}\right) \exp \left(p_{2}^{\prime} x\right)-\left(p_{2}^{\prime}+\alpha_{2}\right) \exp \left(p_{2} x\right)\right]$

avec :

$$
\left.\begin{array}{ll}
2 & p_{2} \\
2 & p_{2}^{\prime}
\end{array}\right\}=-\sum_{1}^{3} \alpha_{i} \pm \Delta_{2}^{1 / 2}
$$

et

$$
\Delta_{2}=\left(\sum_{1}^{3} \alpha_{i}\right)^{2}-4\left(\alpha_{2}\left(\alpha_{1}+\alpha_{3}\right)-\eta_{1} \eta_{2} U_{30}^{2}\right) .
$$
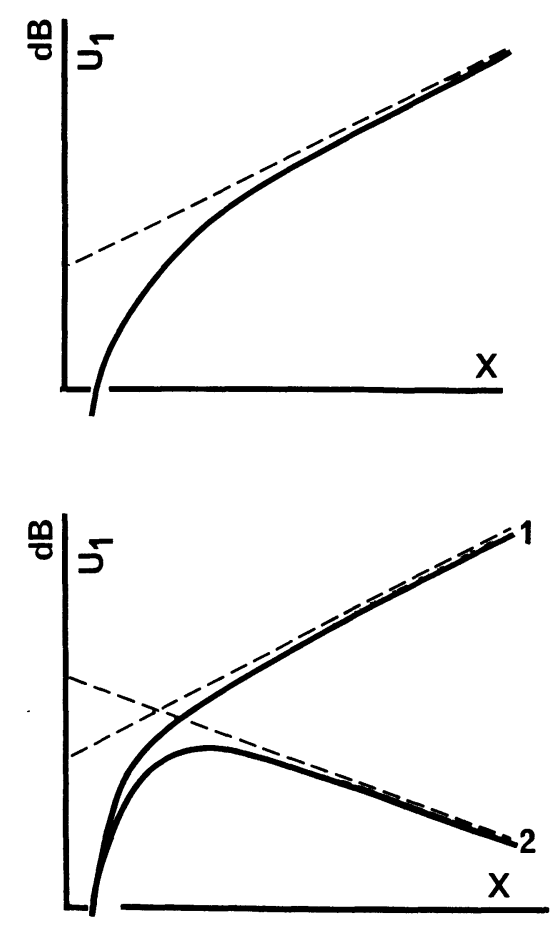

Fig. 2. - Schéma de la variation théorique de $\left.U_{1}(x): a\right)$ en dessus du seuil d'amplification linéaire $; b$ ) en dessous du seuil d'amplification linéaire :

1 pour $U_{30}^{2}>\alpha_{1} \frac{\alpha_{2}+\alpha_{3}}{\eta_{1} \eta_{2}}$ atténuation paramétrique ;

2 pour $U_{30}^{2}>\alpha_{1} \frac{\alpha_{2}+\alpha_{3}}{\eta_{1} \eta_{2}}$ amplification paramétrique.
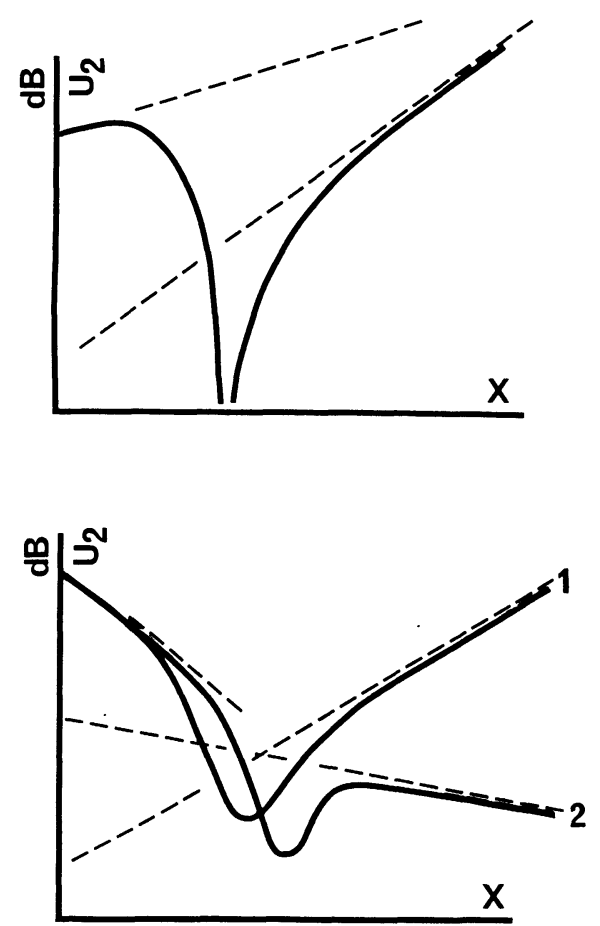

Fig. 3. - Schéma de la variation théorique de $\left.U_{2}(x): a\right)$ en dessus du seuil d'amplification linéaire ; $b$ ) en dessous du seuil d'amplification linéaire :

1 pour $U_{30}^{2}>\alpha_{2} \frac{\alpha_{1}+\alpha_{3}}{\eta_{1} \eta_{2}}$ atténuation paramétrique ;

2 pour $U_{30}^{2}>\alpha_{1} \frac{\alpha_{2}+\alpha_{3}}{\eta_{1} \eta_{2}}$ amplification paramétrique. 
Les variations de $U_{1}$ et $U_{2}$ en fonction de la longueur d'interaction $\mathrm{x}$ sont schématisées sur les figures 2 et 3 .

Lorsque le niveau de la pompe est suffisant (par exemple $\left.U_{30}^{2}>\alpha_{1} \cdot \frac{\alpha_{2}+\alpha_{3}}{\eta_{1} \eta_{2}}\right)$ on obtient une amplification de $U_{1}$ (ou $U_{2}$ ) même en dessous du seuil d'amplification linéaire (Fig. $2 b 1$ et $3 b 1$ ). On peut d'ailleurs prévoir l'existence de deux modes de fonctionnement différents en réécrivant par exemple la première des équations (15):

$$
\frac{\mathrm{d}}{\mathrm{d} x}\left(\operatorname{Ln} U_{1}\right)=-\alpha_{1}+\eta_{1} \frac{U_{2} U_{30}}{U_{1}}
$$

où l'on voit clairement que le second membre peut devenir positif, et donc conduire à une amplification, pour des valeurs suffisantes de $U_{30}$. Cependant la signification physique de la limite entre les 2 modes n'apparaît pas évidente compte tenu des hypothèses simplificatrices adoptées et du fait que l'on opère sur des termes complexes.

Les courbes expérimentales des figures 4 et 5 montrent que l'accord est particulièrement satisfaisant compte tenu de toutes les restrictions imposées dans chaque étape de la mise en équation et de la résolution du problème. La figure $5 a$ n'a pas de branche croissante analogue à celle de la figure $3 a$. Ceci tient au fait que nous n'avons pas une longueur d'interaction suffisante compte tenu de la puissance de pompe dont
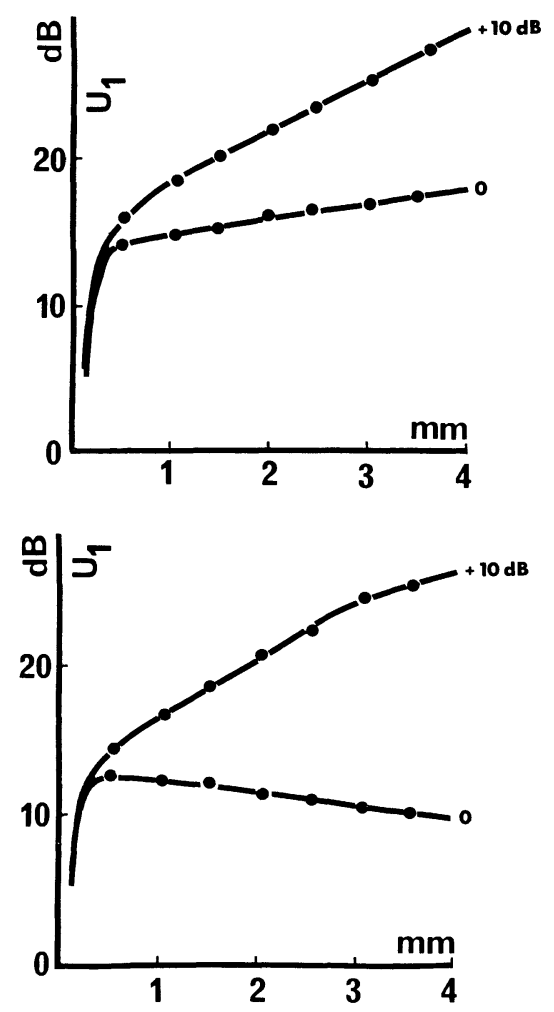

FIG. 4. - Variation expérimentale de $U_{1}$ en fonction de la longueur d'interaction pour deux niveaux de $U_{30}$, l'un supérieur à l'autre de $10 \mathrm{~dB}$. En dessus $(b)$ et en dessous $(b)$ du seuil d'amplification linéaire.

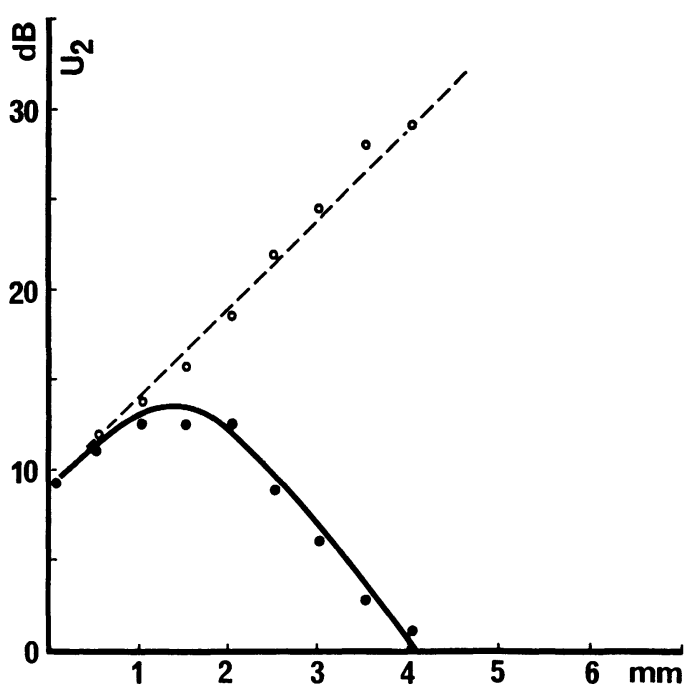

(a)

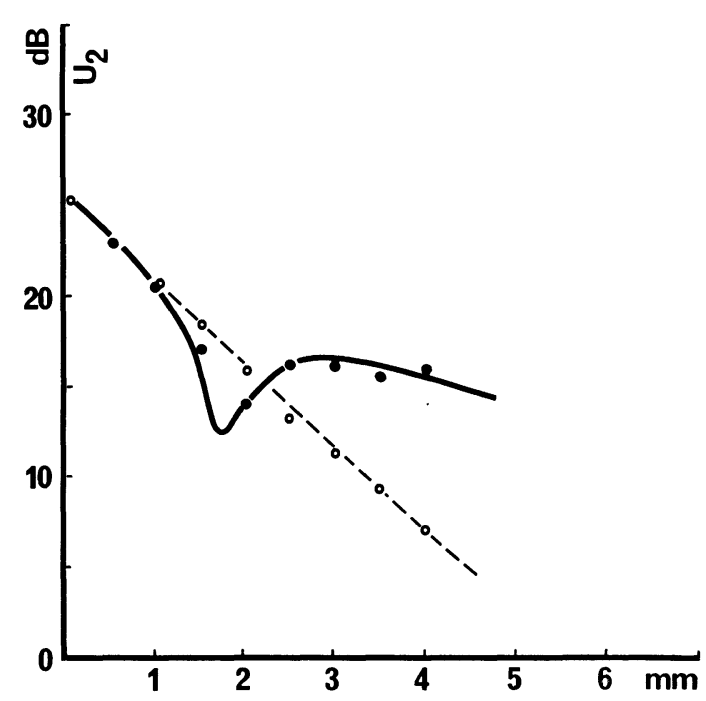

(b)

Fig. 5. - Variation expérimentale de $U_{2}$ en fonction de la longueur d'interaction. En dessus $(a)$ et en dessous $(b)$ du seuil d'amplification linéaire. En pointillé : comportement linéaire de $U_{2}$ c'est-à-dire en l'absence de pompe.

nous disposons. Pour la même raison (niveau insuffisant de $U_{30}$ ) la courbe 1 de la figure $3 b$ n'a pu être observée. Certaines restrictions sont difficilement justifiables, en particulier, pour que la théorie conserve une valeur pour des longueurs d'interaction raisonnables, il faut que $\alpha_{3}$ et $\Delta k$ soient très petits. Cette condition n'est réalisée que pour $E_{0} \simeq V_{\mathrm{s}} / \mu$ c'est-àdire quand, diffusion mise à part, les phénomènes d'interaction électroacoustique sont les plus faibles puisque ondes mécaniques et porteurs se déplacent avec la même vitesse.

3.11 Solution numérique. - Pour étendre le domaine de validité du calcul, nous avons dans une deuxième étape, effectué l'intégration numérique du système (14) dans le cadre de nos conditions expéri- 
mentales, sans aucune hypothèse restrictive, et en tenant compte de la diffusion des porteurs.

L'allure générale des solutions est illustrée sur les figures $6 a, b, c$ et $d$. Elles ont été obtenues par la méthode d'intégration de Runge-Kutta au $4^{\mathrm{e}}$ ordre avec les paramètres usuels du $\mathrm{CdS}$ [6].

L'unité d'amplitude $(\mathrm{O} \mathrm{dB})$ portée sur les courbes calculées est l'angström ce qui correspond pour une onde plane à un flux de puissance de $17 \mathrm{~dB} \mathrm{~m} / \mathrm{mm}^{2}$.

Ces figures montrent que :

- les formes des courbes représentant les amplitudes $U_{1}$ et $U_{2}$ en fonction de la longueur d'interaction sont très ressemblantes à celles obtenues à partir de la solution analytique ;

- l'effet de modulation due au terme $\Delta k$ apparaît sur la figure $6 d$;

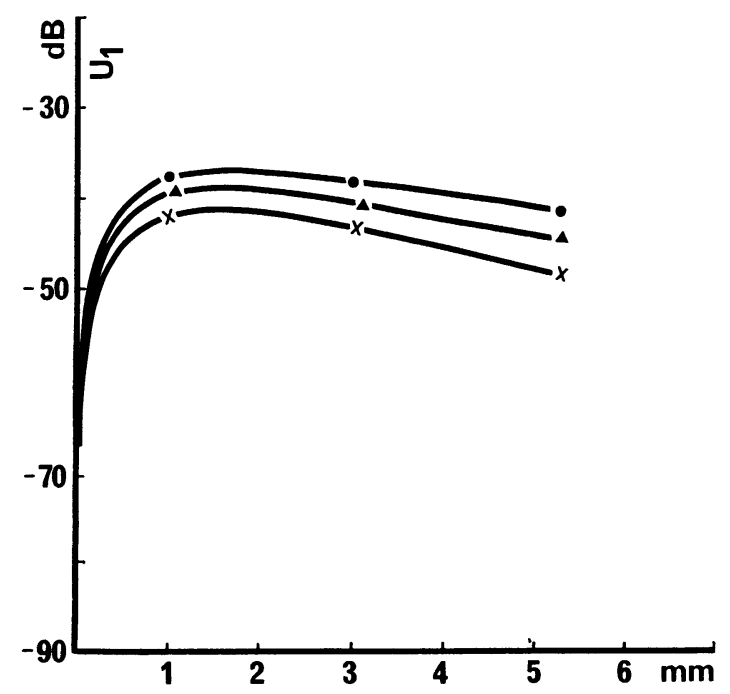

(a)

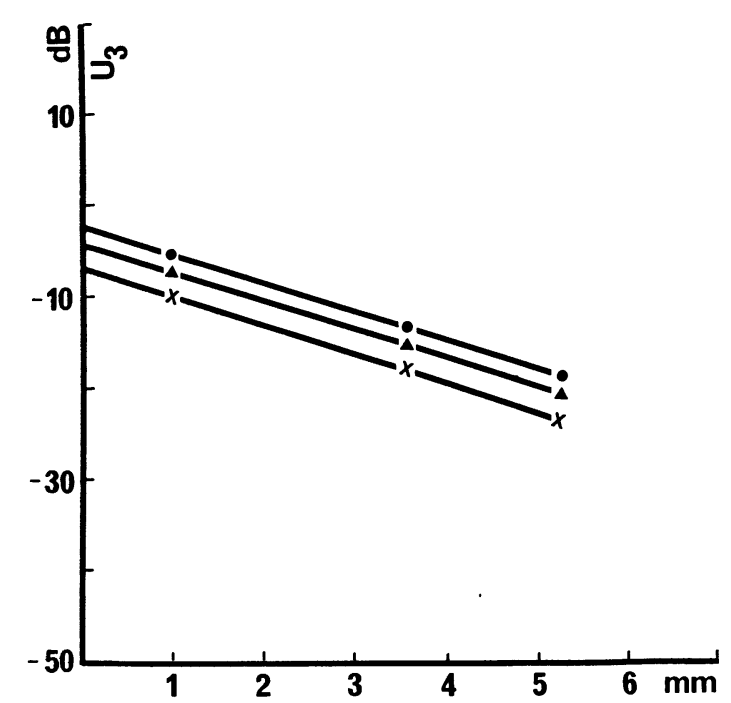

(c)
- $U_{3}$ n'est pas perturbée par la présence des termes d'ordre 2 ;

- $U_{1}$ atteint rapidement le niveau de $U_{2}$ et peut même devenir supérieure ;

- $U_{4}$ est toujours inférieure à $U_{1}$, de 10 à $15 \mathrm{~dB}$.

Cette dernière remarque et celle concernant $U_{3}$ justifient à posteriori 2 hypothèses faites dans la recherche de la solution analytique.

- En dessous du seuil toutes les courbes calculées représentent de façon très satisfaisante les résultats de nos expériences, figures $6 b$ et 7 . On remarque en particulier le déplacement vers les $x$ croissants du minimum de $U_{2}$ quand $U_{30}$ diminue et la possibilité d'une amplification paramétrique observée expérimentalement (Fig. 5b).

- L'accord théorie expérience est aussi particu-

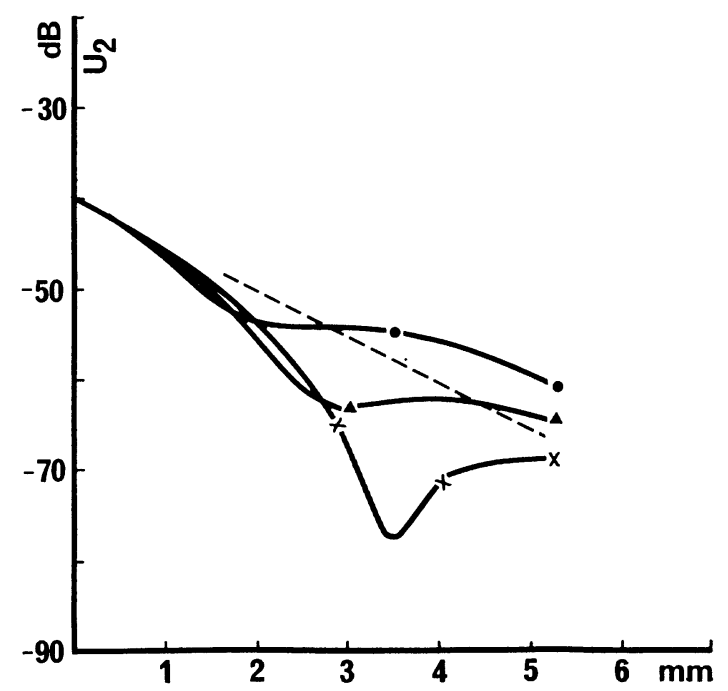

(b)

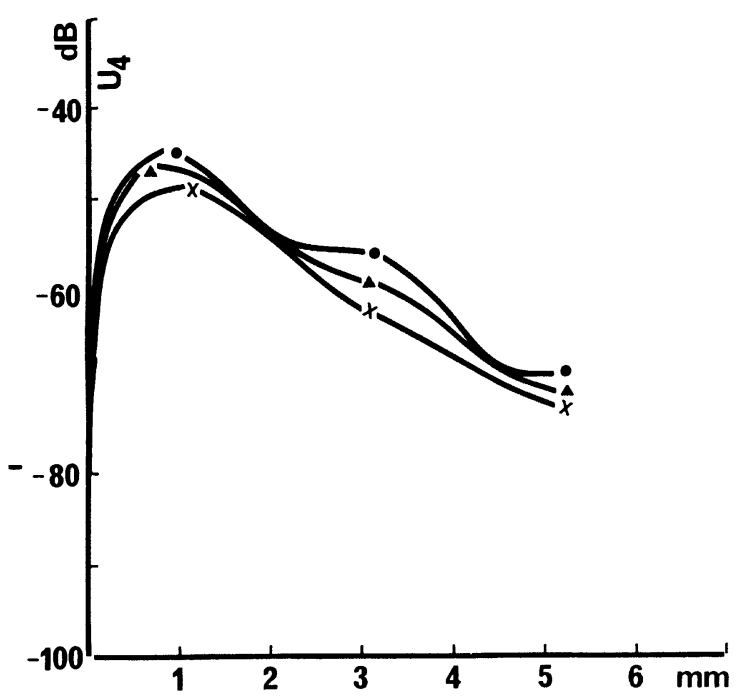

(d)

FIG. 6. - Détermination numérique des variations de : $a, U_{1}(x) ; b, U_{2}(x) ; c, U_{3}(x)$ et $d, U_{4}(x)$ pour 4 valeurs du niveau de $U_{30} . \times, U_{30}=0,45 \AA ; \Delta, U_{30}=060 \AA ; 0, U_{30}=0,75 \AA$ et courbe en pointillés $U_{30}=0$. Ces courbes montrent l'effet paramétrique sur $U_{2}, U_{1}$ et $U_{4}$ et le comportement conforme à la théorie linéaire de $U_{3}$.

$$
\gamma=0,05 \omega_{\mathrm{c}}=5 \times 10^{7} \mathrm{rad} / \mathrm{s} \omega_{\mathrm{D}}=10^{9} \mathrm{rad} / \mathrm{s} .
$$




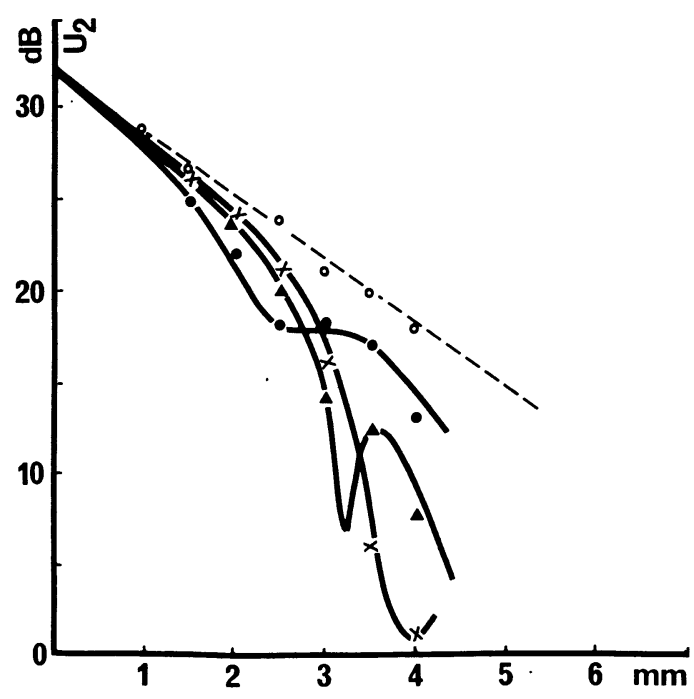

FIG. 7. - Variation expérimentale de $U_{2}$ en fonction de la longueur d'interaction pour 4 niveaux de $U_{3} ; \bullet, U_{30}=15 \mathrm{dBm}$; $\Delta, U_{30}=11 \mathrm{dBm} ; X, U_{30}=10 \mathrm{dBm}$ mettant en évidence le glissement du minimum vers les faibles longueurs d'interaction quand $U_{30}$ augmente.

lièrement satisfaisant lorsqu'on opère à longueur d'interaction constante et qu'on fait varier le niveau d'injection $U_{30}$ de la pompe (Fig. 8 et 9). Notons que l'effet paramétrique n'apparaît sur $U_{2}$ ou $U_{1}$ que lorsque l'amplitude de la pompe devient de l'ordre de l'angström. Pour des niveaux plus faibles de la pompe, chacune des 2 ondes primaires $U_{2}$ et $U_{3}$ se comporte comme si elle se propageait seule dans le cristal. La courbe théorique montre qu'il existe une longueur d'interaction pour laquelle la perturbation sur $U_{2}$ est maximale. La longueur d'interaction dont nous disposons expérimentalement doit être plus courte que cette longueur optimale.

- En dessus du seuil l'accord est encore satisfaisant tant que les tensions d'accélération sont très différentes de celles qui correspondent à l'un des maxima d'amplification linéaire de la pompe du signal $\left(\gamma_{\mathrm{i}}=\omega_{\mathrm{c}} / \omega_{\mathrm{i}}\right)$.

En effet on obtient pour $U_{2}$ une faible atténuation au voisinage immédiat du seuil d'amplification linéaire, et une légère amplification paramétrique pour les grandes tensions d'accélération.

Par contre, autour d'un maximum d'amplification d'une des ondes, ce modèle prévoit une très forte amplification paramétrique de toutes les ondes en interaction alors qu'expérimentalement ceci n'a jamais pû être vérifié.

Ce désaccord ne provient pas de la présence des pièges dans le cristal [8] qui entraîne une dispersion en fréquence des seuils d'amplification linéaire car lorsqu'on simule cet effet en associant à chaque fréquence une mobilité différente, les courbes obtenues conservent une allure très voisine des précédentes.

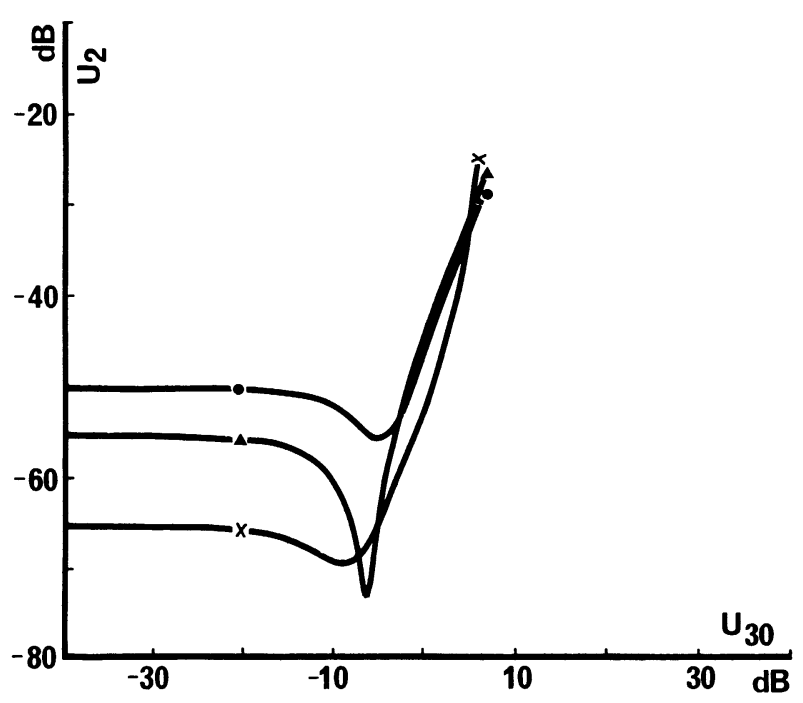

(a)

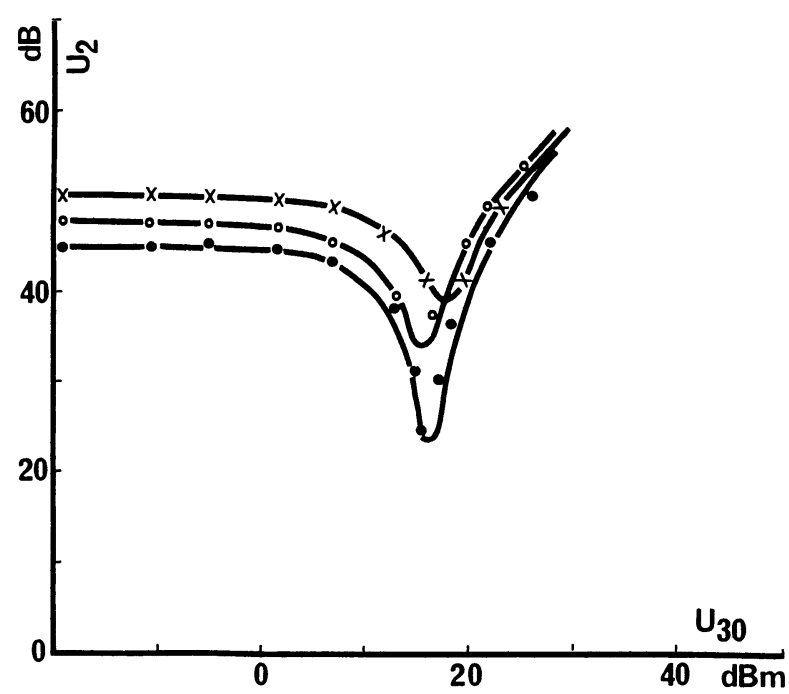

(b)

FIG. 8. - Variation de $U_{2}$ en fonction de $U_{30}$ à longueur d'interaction constante $: a$ ) variation théorique pour 3 longueurs d'interaction : $2 \mathrm{~mm} ; \Delta 3 \mathrm{~mm} ; \times 5 \mathrm{~mm} ; b$ ) variation expérimentale pour $1=: \times 3 \mathrm{~mm} ; \bigcirc, 3,5 \mathrm{~mm} ; 4 \mathrm{~mm}$. Pour les courbes théoriques

$$
\gamma=0,05 \omega_{\mathrm{c}}=5 \times 10^{7} \mathrm{rad} / \mathrm{s} \omega_{\mathrm{D}}=10^{9} \mathrm{rad} / \mathrm{s} .
$$

3.1.2 Effets des harmoniques de rang supérieur. En nous inspirant des théories sur la génération d'harmoniques dans les matériaux passifs [9], nous avons injecté dans le système d'équations (14) les harmoniques 2 des ondes primaires en supposant que les harmoniques 2 de $U_{1}$ et de $U_{4}$ étaient d'un ordre supérieur à ceux de $U_{2}$ et $U_{3}$. A ce niveau, le calcul met donc en jeu 6 ondes : $U_{1}(30 \mathrm{MHz}), U_{2}(70 \mathrm{MHz}), U_{3}(40 \mathrm{MHz})$, $U_{4}(110 \mathrm{MHz}), U_{22}(140 \mathrm{MHz})$ et $U_{33}(80 \mathrm{MHz})$. 


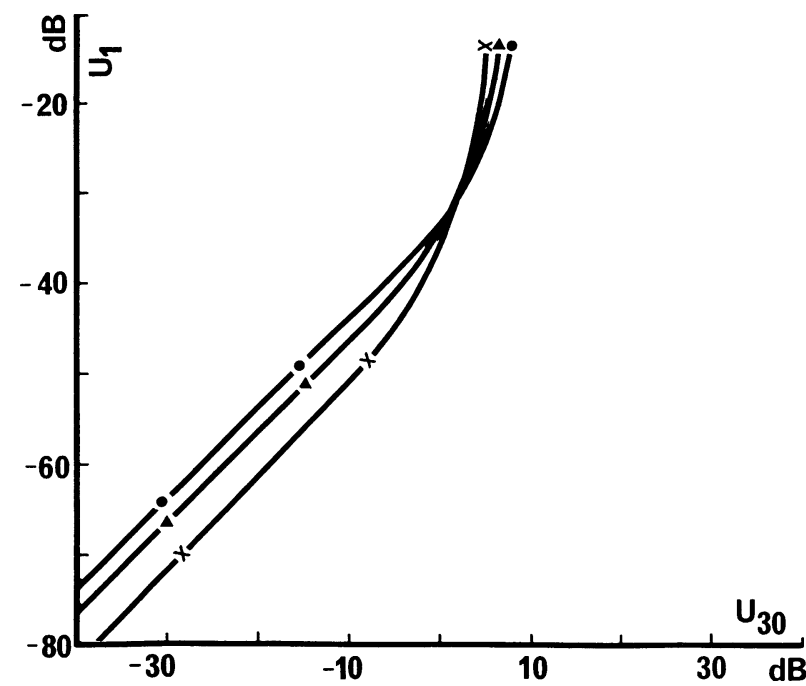

(a)

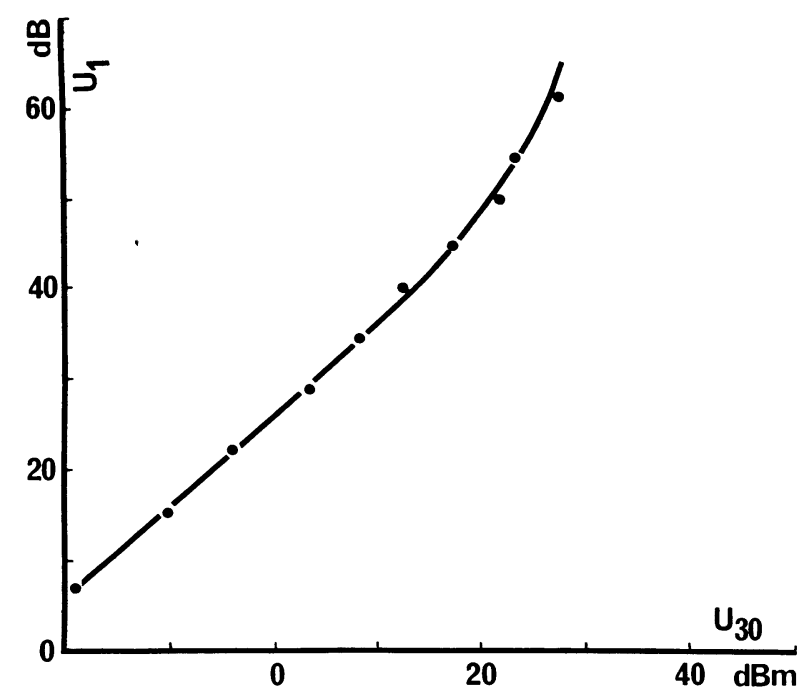

(b)

FIG. 9. - Variation de $U_{1}$ en fonction de $U_{30}$ à longueur d'interaction constante : $a$ ) variation théorique pour 3 longueurs d'interaction $\bigcirc \mathrm{mm} ; \Delta 3 \mathrm{~mm} ; \times 5 \mathrm{~mm} ; b)$ analogue expérimentale pour $1=4 \mathrm{~mm}$. Pour les courbes théoriques

$$
\gamma=0,05 \omega_{\mathrm{c}}=5 \times 10^{7} \mathrm{rad} / \mathrm{s} \omega_{\mathrm{D}}=10^{9} \mathrm{rad} / \mathrm{s} .
$$

L'intégration du nouveau système d'équations différentielles met en évidence la perturbation de $U_{3}$ par son harmonique $2, U_{33}$ (Fig. 10), résultat que nous avons observé expérimentalement (Fig. 11).

4. Conclusion. - A partir des études théoriques existantes $[4,6]$ sur les effets paramétriques en milieu actif nous avons tenté d'apporter une explication à nos résultats expérimentaux.

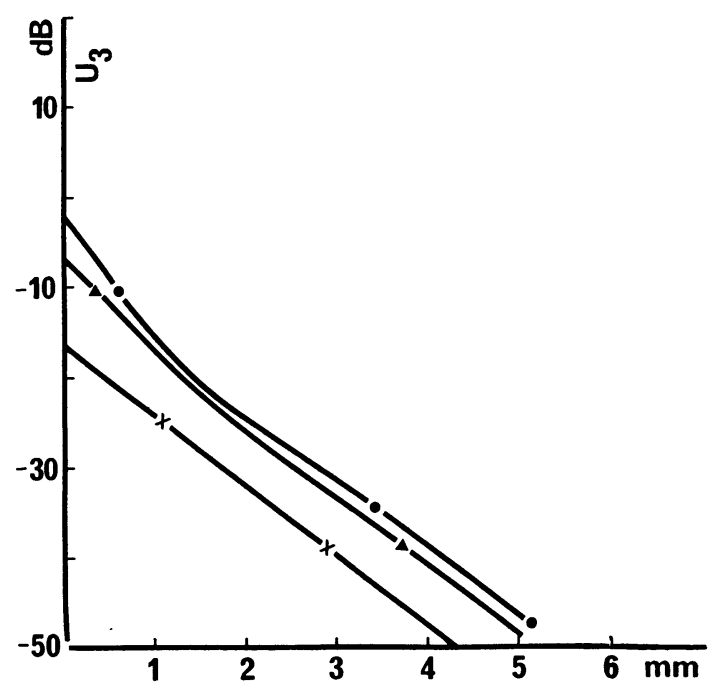

Fig. 10. - Mise en évidence théorique de la déformation de $U_{3}$ par son harmonique 2 pour 3 niveaux de $U_{30}: 0,75 \AA$; $\Delta 0,45 \AA ; \times 0,15 \AA$.

$$
\gamma=0,20 \omega_{\mathrm{c}}=510^{7} \mathrm{rad} / \mathrm{s} \omega_{\mathrm{D}}=10^{9} \mathrm{rad} / \mathrm{s} .
$$

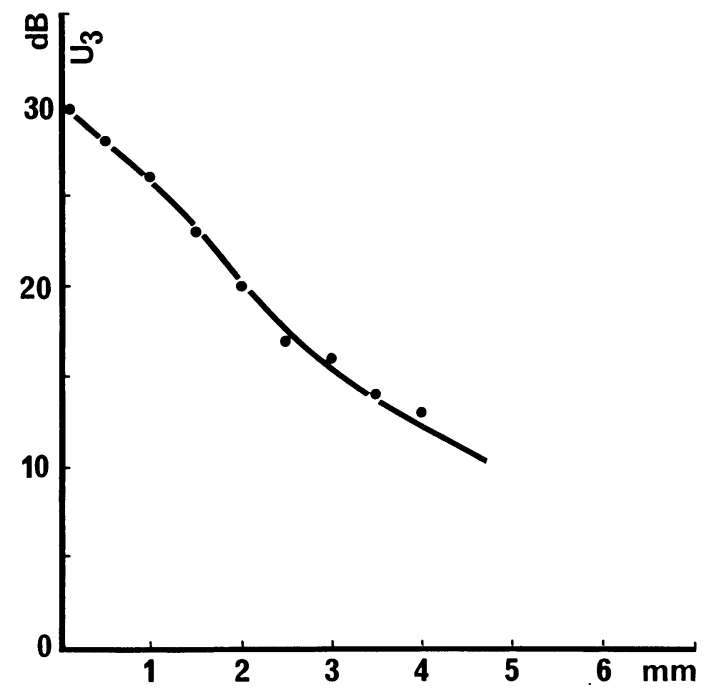

Fig. 11. - Mise en évidence expérimentale de l'effet de l'harmonique 2 sur une onde acoustique, $U_{3}$ pour $U_{30}=36 \mathrm{dBm}$.

Le désaccord observé au voisinage des maxima d'amplification, qui ne peut être expliqué ni par les phénomènes de piégeage ni par l'introduction d'harmoniques de rang 2 des ondes incidentes doit certainement être imputé à la méthode de calcul du champ du deuxième ordre.

Ce champ est en effet calculé par une méthode de perturbation qui ne saurait être rigoureuse lorsque les signaux du deuxième ordre deviennent d'amplitude comparable aux signaux incidents. 


\section{Bibliographie}

[1] Rouzeyre, M., Sagnes, G., Bastide, G., Electron. Lett. 10 (1974) 442-443.

[2] Bleustein, J. F., Appl. Phys. Lett. 13 (1968) 412-413.

[3] Gulayev, Y., Sov. Phys. JETP. Lett. 9 (1969) 63-65.

[4] Hutson, A. R., White, D. L., J. Appl. Phys. 33 (1962) 40-47.

[5] Rouzeyre, M., Cambon, G., Solid. State. Commun. 11 (1972) 1085-1088.
[6] Conwell, E. M., Ganguly, A. K., Phys. Rev. B, 4 (1971) 2535-2557.

[7] Ridley, B. K., J. Phys. C. Solid State Phys. 6 (1973) 16051614.

[8] Uchida, I., Ishiguro, T., Sasaki, Y., Susuki, T., J. Phys. Soc. Japan 19 (1964) 674-680.

[9] Adler, E. L., NAssar, A. A., Ultrasonics Symposium Proceedings IEEE, Sonics, Ultrasonics, (1973) 268-270. 